\title{
BUILDING FLOOR PLAN RECONSTRUCTION FROM SLAM-BASED POINT CLOUD USING RANSAC ALGORITHM
}

\author{
M. Hossein Pouraghdam ${ }^{1}$, M. Saadatseresht ${ }^{1,}$ H. Rastiveis ${ }^{1, *}$, A. Abzal $^{1,}$ M. Hasanlou ${ }^{1}$ \\ ${ }^{1}$ School of Surveying and Geospatial Engineering, College of Engineering, University of Tehran, Tehran; (m.pouraghdam, msaadat, \\ hrasti, ali.abzal, hasanlou)@ut.ac.ir
}

Commission II, WG II/7

KEY WORDS: SLAM, Point Cloud, Segmentation, Floor Plan, Indoor Modeling, RANSAC

\begin{abstract}
:
In recent years, the applications of interior and exterior model of buildings have been increased in the field of surveying and mapping. This paper presents a new method for extracting a two-dimensional (2D) floor plan of a building from Simultaneous localization and mapping (SLAM)-based point clouds. In the proposed algorithm, after preprocessing, the voxel space is generated for the point cloud. Then, the optimal section of the voxel cube to generate building floor plan is identified. Finally, the linear structures and walls are extracted using the random sample consensus (RANSAC) algorithm. The proposed algorithm was examined on a collected point clouds of a building, and the walls of this building were automatically extracted. To evaluate the proposed method, the obtained walls by the algorithm were compared with the manually extracted walls. The algorithm successfully extracted almost $90 \%$ of the walls in the test area. Moreover, the average error of $3 \mathrm{~cm}$ for the extracted walls proved the high accuracy of the proposed method for building floor plan modeling.
\end{abstract}

\section{INTRODUCTION}

Indoor building modeling and floor plan generation are useful in many fields such as protection, documentation, facility management, security, and building information models (BIM) (Previtali et al., 2014). In this regard, 2D floor plans can be applicable in internal navigation, augmented reality and virtualization, and building energy simulation softwares (Turner and Zakhor, 2014). Also, they are applicable for legal map production of complex buildings to evaluate the dimension of rooms and other structural features (Stojanovic et al., 2018).

In the traditional methods for generating these maps they are produced by directly measuting the interior spaces using strips. Since these methods are carried out by interaction of a human operator, measurement in this manner is time-consuming and costly, and therefore, the results may be accompanied by human errors. Therefore, indoor 3D data acquisition technologies based on Simultaneous Localization And Mapping (SLAM) have been developed in terms of proficiency and accuracy (Maboudi et al., 2018). These tehnologies are three-dimensional (3D) mobile mapping technology that scans its environments to produce very accurate and dense $3 \mathrm{D}$ point clouds within minutes, while on the move. Despite the increasing of data collection speed and accuracy, due to the high volume of data, manual processing of these data is still tedious. Therefore, researchers have been focusing on developing automated techniques in order to obtain interior geometric details of buildings. The aim of developing new processing methods are automatic feature extraction and model generation from point cloud, which should be able to process high-density data (Babacan et al., 2016).

There are several challenges for automatic 2D building plan extraction including manipulating large volume point cloud, occluded areas, optimal cross-section of point cloud, recognition and reconstruction of $2 \mathrm{D}$ linear features to provide architectural map. In this paper, a method for automated modelling of linear features from a SLAM-based point cloud as a floor plan of the building is proposed. In the prpoposed method, first a 2D floor plan is produced and then, the architectural map including walls is created.

To simplify our solution, the following assumptions are considered: (1) the point cloud has a high density with common noise level, (2) the point cloud of different stations are geometrically co-registered and consistent, (3) there is not curved surfaces in the internal structure of the building and wall, ceiling and floors are planar, and (4) data from the main building structure is generally completed without big loss. In relation to the problem statement, the following key questions should be answered through our experiments: (1) in presence of room furniture, which method is more suitable for cross-section extraction? (2) what is the optimal value for the parameters of the proposed algorithm for floor plan segment extraction? and (3) what is the geometric accuracy and completeness of the 2D extracted plan?

The structure of the paper is as follows: In Section 2, we describe related work to this research. Section 3 describes our proposed algorithm to generate floor plans. The proposed solution, along with implementation on real data, is presented in Section 4. Finally, in Section 5 results of the proposed algorithm are reported.

\section{RELATED WORK}

For building interior model extraction, line and planar surface is essential. Since the majority of studies about building floor plan

\footnotetext{
* Corresponding author
} 
generation has used line or planar surface modeling in their methods, a review of related workds about this issue is presented. Today, developing point cloud collection technologies and processing methods have been the subject of much interest by researchers. In this context, floor plans can be considered a temporary step to approach future internal building models. Over the years, many algorithms for line modeling from point clouds have been studied by researchers including RANSAC (Hong et al., 2015; Jung et al., 2014; Li et al., 2018; Ochmann et al., 2014; Thomson and Boehm, 2015), Hough Transform (HT) (Dumitru et al., 2013; Huber et al., 2011; Oesau et al., 2013; Okorn et al., 2010), split and merge (Zhang and Ghosh, 2000), Expectation-Maximiation (EM) (Liu et al., 2001). In this case, Nguyen et al. (2007) presented a comprehensive comparison between a number of line extraction algorithms using 2D laser scanner data. Also, a comprehensive comparison between existing algorithms for fitting a flat plate to laser scanner point clouds was presented by Nurunnabi et al. (2014).

Extracting 2D floor plan from point cloud data has also been a signification topic so far. Several methods have been proposed in the recent literature for extracting $2 \mathrm{D}$ floor plan from point cloud data. This section provides an overview of some research on building modeling. Budroni and Böhm (2009) provided a plane sweep algorithm for the segmentation of a point cloud in order to recognize the planar structures of a room. Also, Okorn et al. (2010) proposed an approach based on the use of Hough Transform algorithm for line detection. In addition, in 2011, Huber et al. developed and evaluated a new method for automatic modelling of vertical structures (i.e. walls) and displaying Building Information Model (BIM) from 3D point clouds based on Okron's floor plan modelling algorithm (Huber et al., 2011). Additionally, Oesau et al. (2013) proposed an automated method using HT algorithm to reconstruct permanent structures such as walls, floors, and ceilings from cloud compartments. In contrast, Previtali et al. (2014) presented a new technique to generate a semantic model of the interior of buildings from point clouds.

The reconstruction of interiors is mostly performed by using interactive or semi-automatic or automated approaches (Previtali et al., 2014). Anagnostopoulos et al. (2016) offered a stable method of extraction walls, ceilings and floors that automatically processes point cloud and identifies and categorizes complication. Simultaneously, Babacan et al. (2016) proposed a new method for automatically extracting floor plans by door detection algorithm from raw laser scanner. Recently, Giorgini et al. (2018) presented a new approach to automate the production of the floor plan from point cloud captured by a laser scanners. In their approach, the floor, ceiling and walls have not been considered flat. Similarly, Nikoohemat et al. (2018) proximated graph-based approaches to identify and label permanent structures such as walls, floors and ceilings and stairs from mobile laser scanners. Moreover, Stojanovic et al. (2018) presented a method for generating 2D and 3D approximate planes from the point cloud.

\section{PROPOSED METHOD}

Based on the aforementioned studies it is clear that still continued research is needed for building modeling from point cloud. Therefore, in this paper a new method for building modeling from SLAM-based point cloud is presented. The workflow of the proposed method is shown in Figure 2. As shown in this figure, the algorithm extracts the buiding floor plan from a SLAM-based point cloud in four steps including (1) Floor points selection, (2) Binary voxel generation, (3) Optimal section selection, (4) Linear features extraction. Each step is descriped in detail in the following subsections.

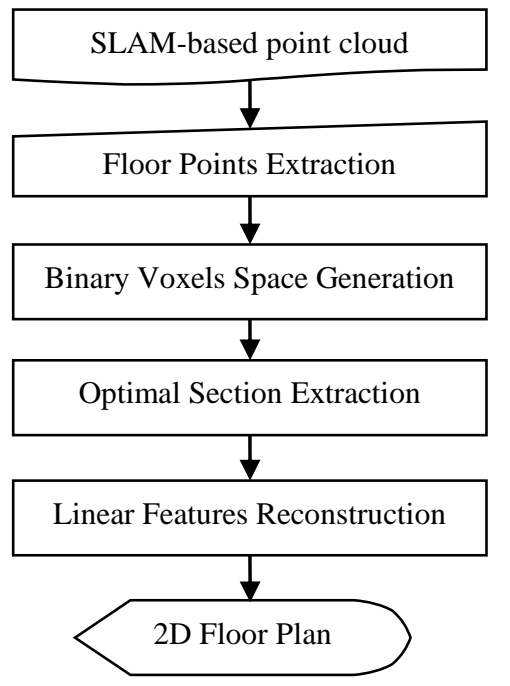

Figure 1. The workflow of the proposed method

\subsection{Floor Points Extraction}

The proposed algorithm extracts a plan for each floor individually. Therefore, in this step, the points of each floor should be extracted to be imported in the processing steps. This helps to simplify the next processes, and is performed manually over the data.

\subsection{Binary Voxel Space Generation}

After selecting the points of a candidate floor, the points are transformed to a regular 3D network called voxel space. If each cell or voxel in this space includes one or more points, it would take 1 ; otherwise, it is an empty of 0 value cell. Since the final voxel space is generated in a binary format, we call it the binary voxel space. Dimensions of the voxels is a challenging parameters which can be determined based on the resolution and density of the point cloud as well as the required final accuracy. Although the smaller cell size would results more accurate 3D voxel space, however, it may increase the redundancy and sparsity of the final space. Conversely, too large cell size may cause information loss, and consequently, less accurate floor plan. Therefore, the cell size should be carefully detected.

\subsection{Optimal Section Extraction}

After the voxel generation of the point cloud, in this stage, we want to find the optimal section that the walls are visible. We create a height histogram. Then, the floor and ceiling are identified from height histogram using the Peak Identification Method presented by Okorn et al. (2010). So, the optimal section is that has the lowest noise (such as room furniture, lamps, computer, etc.), has no occluded area, and the overall structure of walls are visible. Also, the optimal section is the most similar to the ground truth wall segments. Therefore, to find the optimal planimetric section of the voxel point cloud, two ideas are studied and performed in the 2D binary raster space: (a) using median along the vertical axis of voxels and (b) using a constant offset distance below ceil. 


\subsection{Linear Features Reconstruction}

The generated raster image is used to reconstruct the linear features of the building floors. For this purpose, powerful line extraction techniques such as HT and RANSAC may be useful. In this research, the RANSAC as a fast and robust approach which can provide more accurate linear parameters is used in order to identify linear structures in that raster image.

The proposed RANSAC algorithm firstly selects two random points from the voxel points, then fits the lines by examining the other points and analyzing same parameters. This process continues until all the points are examined. Our algorithm is a function of distance between the point of line. Then, it assumes a line that passes through these two selected points. Then, calculates the number of pixels which are less than one pixel from the assumed line. Then, obtains the parameters of the line and distance of all points from this hypothetical line. After that, it considers points whose distance is less than one pixel as inliers. The RANSAC algorithm repeatedly fits the best line to our voxel point. At this stage, we want to separate the lines. For this purpose, we introduce two parameters to the algorithm; gap parameter and noise threshold. The gap parameter refers to the maximum allowable distance to merge two disconnected lines, and the noise threshold refers to the smallest acceptable length of recognized lines. At the final step, a line is fitted to each voxel group to reconstruct the linear feature in that voxel group. We did it by a robust line fitting method which uses least square fitting after removing outlier point clouds in each voxels.

\section{TEST AND RESULT}

\subsection{Dataset}

In order to evaluate the proposed algorithm, a point cloud dataset was collected from central building of the College of Engineering, the University of Tehran, Iran. The data was gathered by GeoSLAM (Zeb-Revo) with the range of $30 \mathrm{~m}$ and $15 \mathrm{~m}$ for interior and exterior, respectively. Also, the scanning time for interior of the building was 34 minutes that 60 million points gathered. Figure 3 shows the overview of the collected point cloud.

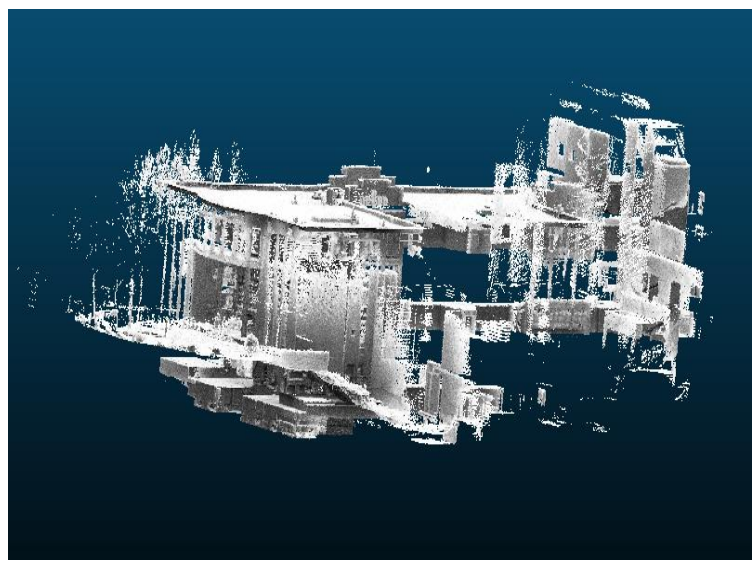

Figure 3. The collected SLAM-based point cloud from the College of Engineering, the University of Tehran.

\subsection{Results}

From the study area, a floor point cloud including about 6 million points was manually extracted at the first step which is depicted in Figure 4.

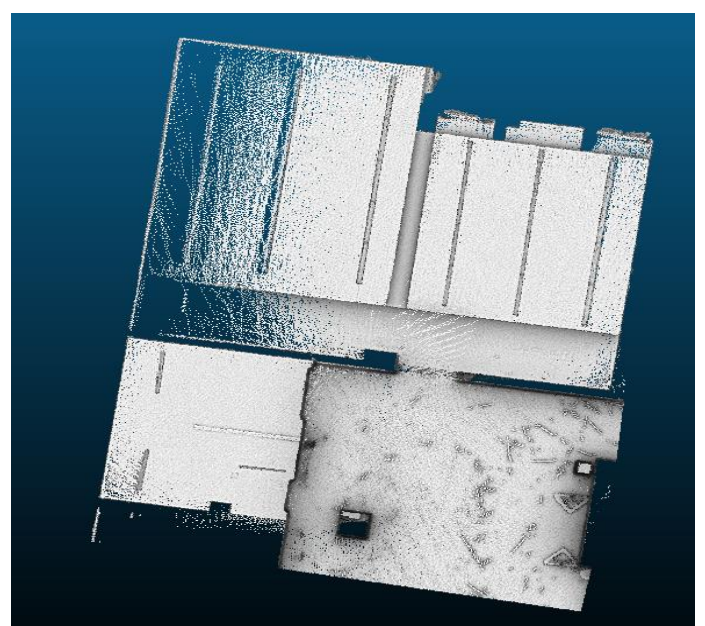

Figure 4. Manually extracted floor points as test data from the College of Engineering dataset.

In our experiments, and based on the resolution of the collected point cloud, we found that the optimum value for voxel size is $0.1 \mathrm{~m}$ in $\mathrm{X}, \mathrm{Y}$, and $\mathrm{Z}$ direction. This value was detected by trial and error. Figure 5 shows the generated Voxel space for the selected floor.

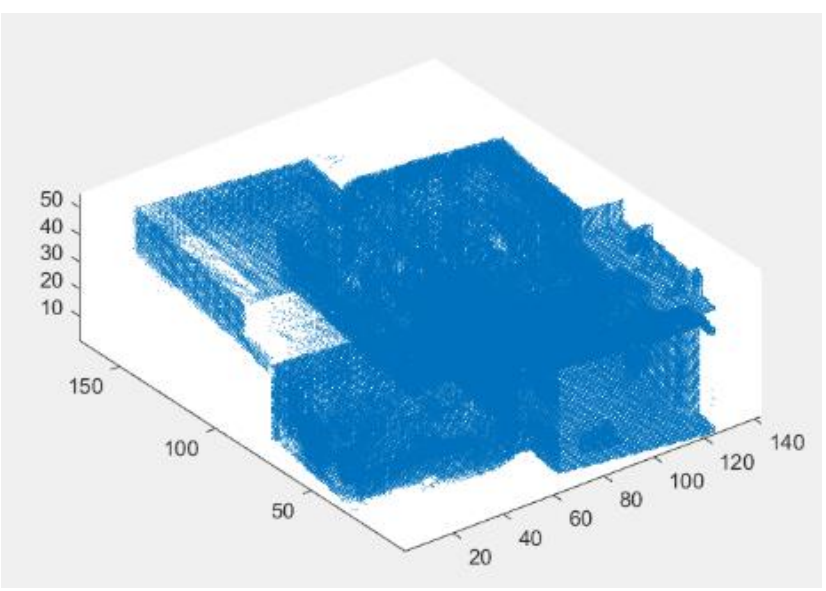

Figure 5 . The binary voxel space considering $0.1 \mathrm{~m}$ voxel size.

For selecting the optimal section, two methods were examined: (a) offset solution, (b) the median solution. As you can see in Figure 6, the offset solution with a distance of $30 \mathrm{~cm}$ below ceil has shown a better output. The extracted 2D points of the best section is shown in Figure 7.

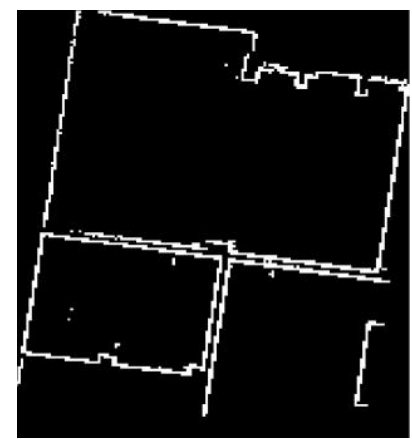

(a)

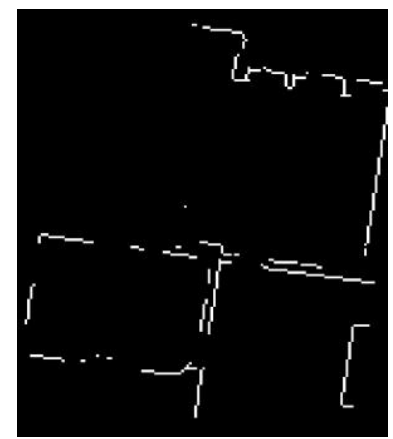

(b)
Figure 6. Optimal section selection results. (a) Extracted sections of the offset solution; (b) the median solution. 


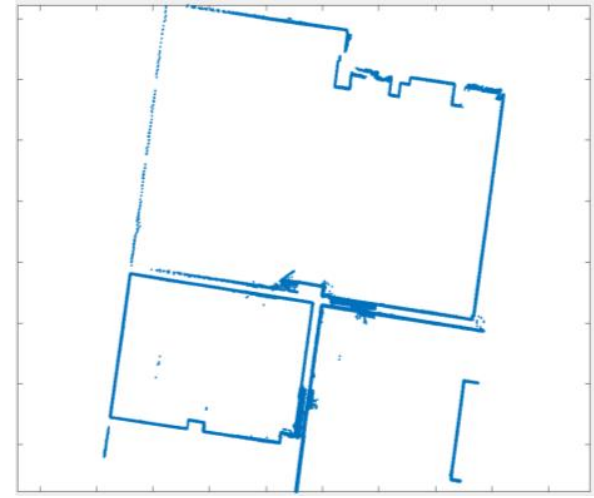

Figure 7. 2D SLAM-based point clouds of the best section

As can be seen in Figure 8, some voxels with similar colors and directions should be grouped into different individual line segments. In addition, some voxels with similar colors and directions should be merged as a single line due to undesirable gaps between them.

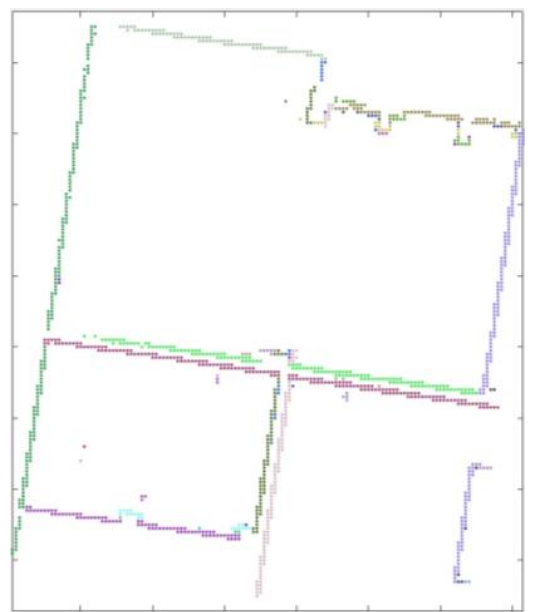

Figure 8. Segmentation of voxels by RANSAC algorithm. Each recognized line has a different color.

In this part, a line is fitted to each voxel group to reconstruct the linear feature in that voxel group. The lines are reconstructed so that two parameters are introduced to the algorithm: gap parameter and noise threshold parameter (Figure 9).

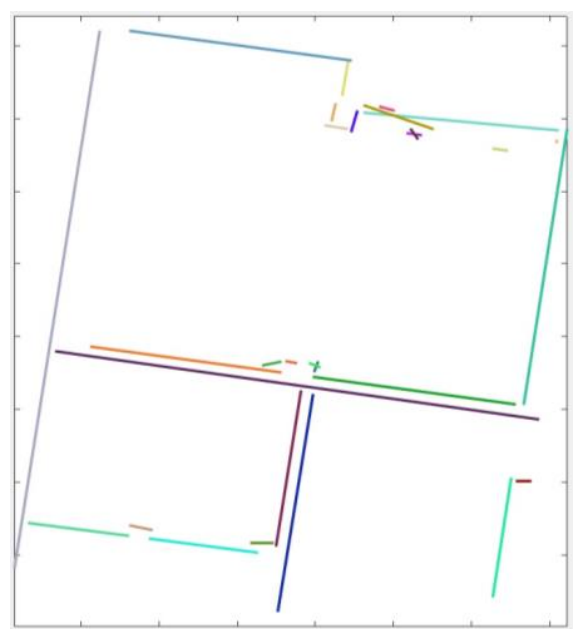

Figure 9. Extracted linear features of the optimal section.

\subsection{Accuracy assessment}

To evaluate the quality of the output segments, they were compared to the ground truth floor plan produced by tracing exact polylines on the test area using a skilled operator. Here, two quality factors of completeness, which measures the ability of linear segment recognition, and the accuracy to measure the quality of the extracted segments were calculated.

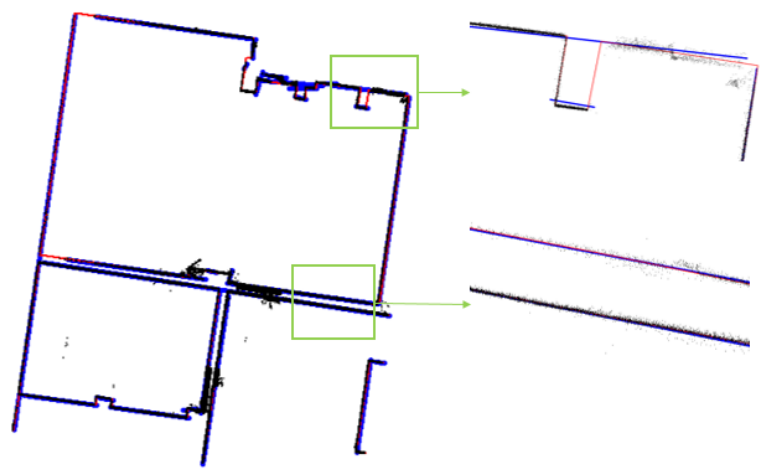

Figure 10. Comparison of the ground truth wall segments (red) and the extracted segments by the proposed algorithm (blue).

Original points of each segment are shown in black.

For completeness evaluation, the total length of all extracted segments was compared to the total length of the ground truth polylines. The length of the reference lines was $94.971 \mathrm{~m}$, while the total length of the extracted segments was $82.580 \mathrm{~m}$. However, $2.974 \mathrm{~m}$ of the segments were lost due to the occluded area. As a result, our proposed algorithm recognized about $90 \%$ of the walls in the dataset.

To evaluate the accuracy of the reconstructed segments, the average distance from the reference polylines were evaluated. The average error of about $3 \mathrm{~cm}$ was seen for the most number of walls, which is a good performance due to the proper voxel size $(0.1 \mathrm{~m})$. Figure 11 illustrates the error distance of the extracted segments and the reference polylines. As can be seen from this figure, the maximum error of $0.2 \mathrm{~m}$ was appeared for segment D16 that may be the sign of linear feature detection error.

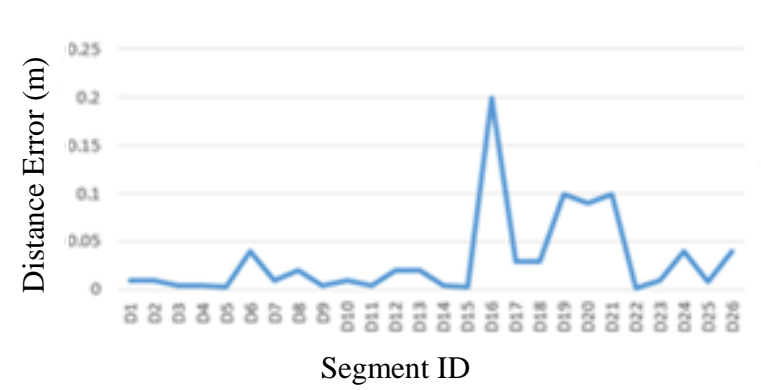

Figure 11. Error distance of the extracted segments and reference polylines.

Because of the random nature of the RANSAC algorithm another experiment was performed to check the reliability of the method. The algorithm was repeated 10 times, and the output was evaluated each time. In this test, the average completeness of $90 \%$ with a sligh difference was reported. 


\subsection{Discussion}

The proposed method has several parameters that should be adjusted; voxel size parameter, gap parameter, noise threshold. In order to find the optimal voxel size, different numbers were examined. By increasing the voxel size, in the lines reconstruction stage, some lines were missed. On the other hand, by increasing gap parameter, the lines were reconstructed in a wrong way. Apart from this and as a matter of fact, increasing the noise threshold parameter to remove small lines leading to losing the longer lines which might be reconstructed correctly.

Therefore, a series of experiments were performed to determine the effect of each parameter on the performance of the algorithm. The optimal voxel size parameter entered by the user into the algorithm was assumed to be $10 \mathrm{~cm}$ during the investigation, and the optimal gap parameter in RANSAC algorithm was 5 voxels. Also, the optimal noise threshold in RANSAC algorithm which refers to the smallest acceptable length of recognized lines is 3 voxels. In fact, lines with less than 3 voxels were considered as noise and were eliminated.

Besides, the proposed RANSAC algorithm has many advantages such as; easy-to-implement procedure, low computation, fast in handling huge data, robust against outliers, flexible in dealing with different structures, and high reliable of its results. However, manually floor points separation step is one of its drawback which reduce the level of automation, and should be considered in furture studies.

\section{CONCLUSION}

In this research, a new method for extracting a 2D floor plan of a building from a SLAM-based point cloud was presented. The proposed algorithm extract a building floor plan in four steps of floor points selection, binary voxel generation, optimal section selection, and linear features extraction. The method was tested on a collected point cloud using GeoSLAM sensor, and $90 \%$ of the average completeness was reported. Using the RANSAC method to recognize line segments and working with one optimal section may be the main advantages of the proposed method.

In fact, recognition and reconstruction of linear segments are two different subjects, and we achieved a fairly good approximation in this study. But to improve the result, we want to reconstruct the linear segments based on original point cloud via an optimization process which gives much higher accuracy than 2D grid points.

\section{Acknowledgements}

The authors would like to thank Nema Pardaz Rayaneh (NPR) company for providing the experimental point cloud data.

\section{REFERENCES}

Anagnostopoulos, I., Pătrăucean, V., Brilakis, I., Vela, P., 2016. Detection of Walls, Floors, and Ceilings in Point Cloud Data, Construction Research Congress 2016, pp. 2302-2311.

Babacan, K., Jung, J., Wichmann, A., Jahromi, B., Shahbazi, M., Sohn, G., Kada, M., 2016. Towards Object Driven Floor Plan Extraction from Laser Point Cloud. International Archives of the Photogrammetry, Remote Sensing \& Spatial Information Sciences 41.

Budroni, A., Böhm, J., 2009. Toward Automatic Reconstruction of Interiors from Laser Data. Proceedings of Virtual Reconstruction and Visualization of Complex Architectures (3D-Arch), 36.

Dumitru, R., Borrmann, D., Nüchter, A., 2013. Interior Reconstruction Using the 3D Hough Transform. Proceedings international archives of the photogrammetry, remote sensing and spatial information sciences 5, W1.

Giorgini, M., Aleotti, J., Monica, R., 2018. Floorplan Generation of Indoor Environments From Large-Scale Terrestrial Laser Scanner Data. IEEE Geoscience and Remote Sensing Letters 16, 796-800.

Hong, S., Jung, J., Kim, S., Cho, H., Lee, J., Heo, J., 2015. Semi-automated Approach to Indoor Mapping for 3D As-Built Building Information Modeling. Computers, Environment and Urban Systems 51, 34-46.

Huber, D., Akinci, B., Oliver, A.A., Anil, E., Okorn, B.E., Xiong, X., 2011. Methods for Automatically Modeling and Representing as-built Building Information Models, Proceedings of the NSF CMMI Research Innovation Conference.

Jung, J., Hong, S., Jeong, S., Kim, S., Cho, H., Hong, S., Heo, J., 2014. Productive Modeling for Development of As-Built BIM of Existing Indoor Structures. Automation in Construction 42, 68-77.

Li, L., Su, F., Yang, F., Zhu, H., Li, D., Zuo, X., Li, F., Liu, Y., Ying, S., 2018. Reconstruction of Three-Dimensional (3D) Indoor Interiors with Multiple Stories via Comprehensive Segmentation. Remote Sensing 10, 1281.

Liu, Y., Emery, R., Chakrabarti, D., Burgard, W., Thrun, S., 2001. Using EM to Learn 3D Models of Indoor Environments with Mobile Robots, ICML, pp. 329-336.

Maboudi, M., Bánhid, D., Gerke, M., 2018. Investigation of Geomatric Performance of an Indoor Mobile Mapping System International Archives of the Photogrammetry, Remote Sensing \& Spatial Information Sciences 42.

Nguyen, V., Gächter, S., Martinelli, A., Tomatis, N., Siegwart, R., 2007. A Comparison of Line Extraction Algorithms Using 2D Range Data for Indoor Mobile Robotics. Autonomous Robots 23, 97-111.

Nikoohemat, S., Peter, M., Oude Elberink, S., Vosselman, G., 2018. Semantic Interpretation of Mobile Laser Scanner Point Clouds in Indoor Scenes Using Trajectories. Remote sensing $10,1754$.

Nurunnabi, A., Belton, D., West, G., 2014. Robust Statistical Approaches for Local Planar Surface Fitting in 3D Laser Scanning Data. ISPRS journal of photogrammetry and Remote Sensing 96, 106-122.

Ochmann, S., Vock, R., Wessel, R., Tamke, M., Klein, R., 2014. Automatic Generation of Structural Building Descriptions from 3D Point Cloud Scans, 2014 International Conference on 
Computer Graphics Theory and Applications (GRAPP). IEEE, pp. 1-8.

Oesau, S., Lafarge, F., Alliez, P., 2013. Indoor Scene Reconstruction Using Primitive-Driven Space Partitioning and Graph-cut.

Okorn, B., Xiong, X., Akinci, B., Huber, D., 2010. Toward Automated Modeling of Floor Plans, Proceedings of the symposium on 3D data processing, visualization and transmission.

Previtali, M., Barazzetti, L., Brumana, R., Scaioni, M., 2014. Towards Automatic Indoor Reconstruction of Cluttered Building Rooms from Point Clouds. ISPRS Annals of Photogrammetry, Remote Sensing \& Spatial Information Sciences 2.

Stojanovic, V., Trapp, M., Richter, R., Döllner, J., 2018. Generation of Approximate 2D and 3D Floor Plans from 3D Point Clouds.

Thomson, C., Boehm, J., 2015. Automatic Geometry Generation from Point Clouds for BIM. Remote Sensing 7, 11753-11775.

Turner, E., Zakhor, A., 2014. Floor Plan Generation and Room Labeling of Indoor Environments from Laser Range Data, 2014 International Conference on Computer Graphics Theory and Applications (GRAPP). IEEE, pp. 1-12.

Zhang, L., Ghosh, B.K., 2000. Line Segment Based Map Building and Localization Using 2D Laser Rangefinder, Proceedings 2000 ICRA. Millennium Conference. IEEE International Conference on Robotics and Automation. Symposia Proceedings (Cat. No. 00CH37065). IEEE, pp. 25382543. 